PREPARED FOR THE U.S. DEPARTMENT OF ENERGY, UNDER CONTRACT DE-AC02-76CH03073

PPPL-3775

PPPL-3775

UC-70

\title{
Precession of Toroidally Passing Particles in Tokamaks and Spherical Tori
}

by

Ya.I. Kolesnichenko, R.B. White, and Yu.V. Yakovenko

January 2003

M

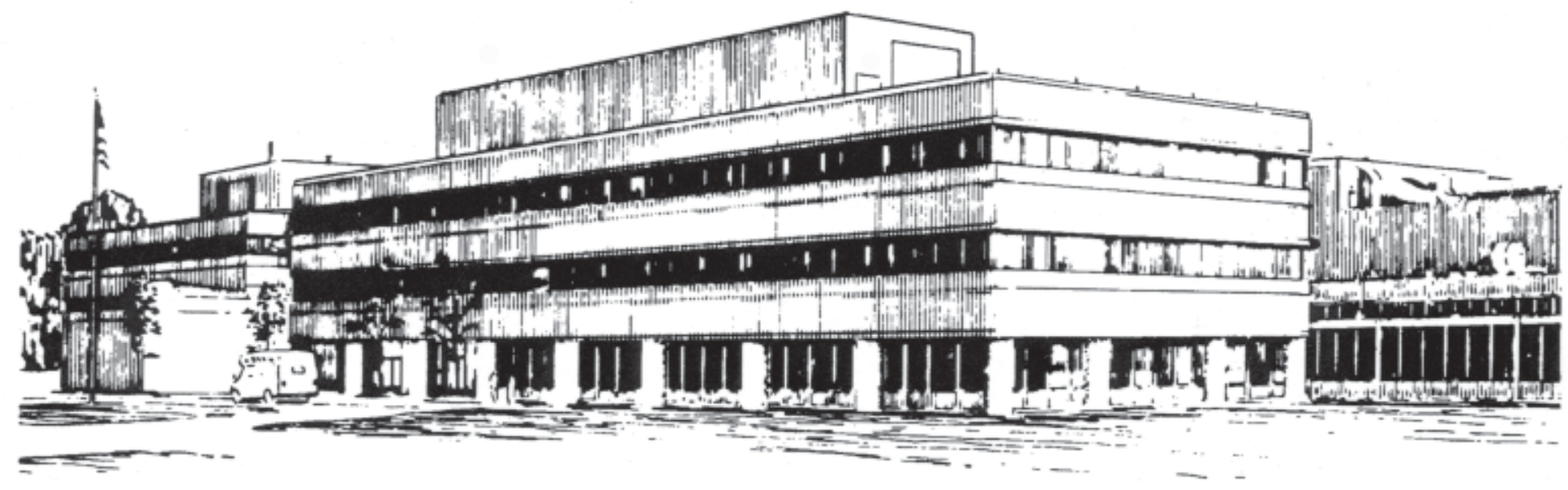

PRINCETON PLASMA PHYSICS LABORATORY PRINCETON UNIVERSITY, PRINCETON, NEW JERSEY 


\section{PPPL Reports Disclaimer}

This report was prepared as an account of work sponsored by an agency of the United States Government. Neither the United States Government nor any agency thereof, nor any of their employees, makes any warranty, express or implied, or assumes any legal liability or responsibility for the accuracy, completeness, or usefulness of any information, apparatus, product, or process disclosed, or represents that its use would not infringe privately owned rights. Reference herein to any specific commercial product, process, or service by trade name, trademark, manufacturer, or otherwise, does not necessarily constitute or imply its endorsement, recommendation, or favoring by the United States Government or any agency thereof. The views and opinions of authors expressed herein do not necessarily state or reflect those of the United States Government or any agency thereof.

\section{Availability}

This report is posted on the U.S. Department of Energy's Princeton Plasma Physics Laboratory Publications and Reports web site in Fiscal Year 2003. The home page for PPPL Reports and Publications is: http://www.pppl.gov/pub_report/

DOE and DOE Contractors can obtain copies of this report from:

U.S. Department of Energy

Office of Scientific and Technical Information

DOE Technical Information Services (DTIS)

P.O. Box 62

Oak Ridge, TN 37831

Telephone: (865) 576-8401

Fax: (865) 576-5728

Email: reports@adonis.osti.gov

This report is available to the general public from:

National Technical Information Service

U.S. Department of Commerce

5285 Port Royal Road

Springfield, VA 22161

Telephone: $1-800-553-6847$ or

(703) $605-6000$

Fax: (703) 321-8547

Internet: http://www.ntis.gov/ordering.htm 


\title{
Precession of toroidally passing particles in tokamaks and spherical tori
}

\author{
Ya.I. Kolesnichenko ${ }^{a}$, R.B. White $^{b}$, Yu.V. Yakovenko ${ }^{a}$ \\ ${ }^{a}$ Institute for Nuclear Research, Prospect Nauky 47, Kyiv, 03680, Ukraine \\ ${ }^{b}$ Princeton Plasma Physics Laboratory, P.O. Box 451, Princeton, NJ, 08543, USA
}

(January 27, 2003)

\begin{abstract}
The toroidal precession of the well-circulating particles and particles that are passing toroidally but trapped poloidally is studied. Expressions for the precession frequency, which are convenient for practical use, are obtained and analyzed. It is found that the key parameters that determine the magnitude and the direction of the precession velocity are the plasma elongation, the magnitudes and profiles of the safety factor and $\beta$ defined as the ratio of the local plasma pressure to the magnetic field pressure at the magnetic axis. An important role of the "paramagnetic" precession in highly elongated plasmas is revealed. The analysis carried out is based on the obtained expressions for the equilibrium magnetic field strength and the field line curvature.
\end{abstract}




\section{INTRODUCTION}

The toroidal precession in axisymmetric toroidal plasmas is the bounce/transit-time-averaged drift motion of the particles in the toroidal direction. Being associated with the toroidal drift, the precession is the most pronounced for the energetic particles. The well-trapped particles have the largest precession velocity because their bounce-averaged drift velocity in the poloidal direction is maximum: $\left\langle v_{d, p o l}\right\rangle \approx v_{d}$, where $v_{d, p o l} \approx-v_{d} \cos \theta$ is the poloidal component of the

toroidal drift velocity $\vec{v}_{d}$, the brackets mean bounce averaging. In contrast to this, $\left\langle v_{d, p o l}\right\rangle \ll v_{d}$ for the well-circulating particles. This implies that the effect of the drift motion on circulating particles strongly decreases the precession frequency and complicates its calculation. On the other hand, the precession of circulating particles is enhanced in high- $\beta$ plasmas of spherical tori by the large Shafranov shift. ${ }^{1}$ This considerably affects both the stability of the plasmas with circulating energetic ions and the transport of energetic ions in magnetohydrodynamically (MHD) active plasmas with high $\beta^{1,2}$

The toroidal precession of trapped particles is studied rather well (see, e.g., Ref. ${ }^{3}$, where a low- $\beta$ plasma was considered, and Ref. ${ }^{4}$, where effects of high $\beta$ were taken into account). The precession of circulating particles is studied much less, especially, in high- $\beta$ plasmas. No papers are published on the precession of the particles passing toroidally but trapped poloidally ("semitrapped" particles), which may constitute a considerable fraction of an energetic ion population in a spherical torus. This motivated the fulfillment of the present work, where the precession of circulating and semi-trapped particles is studied.

The structure of the work is as follows. In Sec. II the precession of well-circulating particles with negligible magnetic moment is considered on the assumptions that the particle orbit width, $\Delta r_{b}$, is small $\left(\Delta r_{b} \ll r\right.$, where $r$ is the radial coordinate) and the plasma cross section is circular. The precession of similar particles moving in the near-axis region of a plasma with a non-circular cross section is studied in Sec. III. In addition, in this section the precession of near-axis circulating particles with arbitrary $\Delta r_{b} / r$ and semi-trapped particles with the small magnetic moment is 
considered. The summary and the conclusions drawn are presented in Sec. IV. Appendix A contains the derivation of a component of the magnetic field line curvature, which is required for the calculation of the precession frequency. In Appendix B, the equilibrium of a non-circular plasma in the paraxial approximation is analyzed, and the magnetic field strength near the magnetic axis is determined.

\section{PRECESSION OF CIRCULATING PARTICLES WITH NARROW ORBITS}

We proceed from the following equations of the particle guiding center motion:

$$
\begin{gathered}
\dot{\theta}=v_{\|} b^{\theta}+v_{d}^{\theta}, \\
\dot{\varphi}=v_{\|} b^{\varphi},
\end{gathered}
$$

where $v_{\|}$is the particle longitudinal velocity,

$$
v_{d}^{\theta}=\frac{1}{\omega_{B}} B^{\varphi} b_{\varphi}\left(v_{\|}^{2} \kappa_{\psi}+\frac{\mu}{M} \frac{\partial B}{\partial \psi}\right)
$$

the Boozer coordinates ${ }^{5} \psi, \theta$ and $\varphi$ are used, the corresponding superscripts/subscripts denote the contra-/co-variant vector components, $\vec{B}$ is the magnetic field, $\vec{b}=\vec{B} / B$, $\vec{\kappa}$ is the field line curvature, $\mu$ is the particle magnetic moment, $\omega_{B}=e B /(M c), M$ and $e$ are the particle mass and charge, respectively.

It follows from Eqs. (1) and (2) that

$$
\langle X\rangle=\omega_{b} \oint_{0}^{2 \pi} \frac{d \theta}{2 \pi} \frac{X}{\left|v_{\|} b^{\theta}+v_{d}^{\theta}\right|}, \text { and } \tau_{b}=\oint_{0}^{2 \pi} \frac{d \theta}{\left|v_{\|} b^{\theta}+v_{d}^{\theta}\right|}
$$

where the integrals are taken along the particle orbits, $\tau_{b}$ is the transit time, $\omega_{b}=2 \pi / \tau_{b}$ is the transit frequency. Using Eq. (4), we obtain:

$$
\langle\dot{\varphi}\rangle=\sigma_{v} \omega_{b} \bar{q}+\omega_{D}
$$

where $\bar{q}=\oint q d \theta /(2 \pi)$, and 


$$
\omega_{D}=-\left\langle q v_{d}^{\theta}\right\rangle
$$

According to Eq. (5), the toroidal motion consists of the motion along the field lines at a flux surface where the safety factor equals to $\bar{q}$ and the average drift motion with the frequency $\omega_{D}$, which we will refer to as the precession frequency. However, this definition of the precession frequency is not unique; e.g., it is possible to choose any other flux surface crossed by the particle during its orbital motion instead of the $q=\bar{q}$ surface as a reference surface. It is clear that the difference between the magnitudes of $\omega_{D}$ defined in different ways can be considerable when the magnetic shear is not small and the orbit width is sufficiently large. However, different definitions of the precession frequency do not affect $\langle\dot{\varphi}\rangle$, which is the only important (for the plasma stability and the particle transport) quantity associated with the average particle motion in the toroidal direction.

Let us calculate $\omega_{D}$ for the particles with the vanishing magnetic moment $(\mu=0)$ in a plasma with a circular cross section. In this case the component $\kappa_{\psi}$ required to know $v_{d}^{\theta}$ can be written as follows, see Appendix A:

$$
\kappa_{\psi}=-\frac{1}{B_{0} r R}\left[\frac{\partial R}{\partial r}+\frac{\epsilon}{q^{2}}+\mathcal{O}\left(\epsilon^{2}\right)\right]
$$

where $\epsilon=r / R_{0}, R_{0}$ is the radius of the magnetic axis, the radial coordinate $r$ is defined by $\psi(r)=B_{0} r^{2} / 2$ with $B_{0}$ the magnetic field at the magnetic axis, and $R=R(r, \theta)$ is the distance from the major axis of the torus. As shown in Appendix A, $R(r, \theta)$ is given by

$$
R=R_{0}\left\{1+\epsilon \cos \theta-\Delta(r) / R_{0}+0.5 \epsilon\left(\Delta^{\prime}+\epsilon\right)[\cos (2 \theta)-1]+\mathcal{O}\left(\epsilon^{3}\right)\right\}
$$

where $\Delta(r)$ is the Shafranov shift, $\Delta \geq 0$ with $\Delta(0)=0, \Delta^{\prime}=d \Delta / d r$. Note that this expression for $R(r, \theta)$ coincides with the corresponding expressions of Refs. ${ }^{6,1}$ because the coordinates used in those works are equivalent to the Boozer coordinates in the considered approximation (see Appendix A for details).

The first and second terms in Eq. (7) describe the contribution to the curvature of the toroidal field and the poloidal field, respectively. The second term does not depend on plasma pressure 
and takes into account that $B R \neq$ const because of plasma paramagnetism. Therefore, we will refer to the contribution of this term to the precession as the "paramagnetic precession".

Using Eqs. (3) and (7), we write the poloidal contravariant component of the drift velocity as

$$
v_{d}^{\theta}=-\frac{\rho v}{r R}\left(\frac{\partial R}{\partial r}+\frac{\epsilon}{q^{2}}\right)
$$

where $\rho=v / \omega_{B}$. We consider circulating particles with narrow orbits. Therefore, we have $r=r_{*}+\Delta_{b}$, where $\Delta_{b} \approx \sigma_{v} q \rho \cos \theta$ with $\sigma_{v}=\operatorname{sgn} v_{\|}$is the orbit half-width and $r_{*}$ is the radius where $\theta=\pi / 2$. One can see that in this case $v_{d}^{\theta} / v_{\|} b^{\theta} \approx-\Delta_{b} / r_{*}$. Taking this into account and noting that $b^{\theta} q R=1+O\left(\epsilon^{2}\right)$ and $q=q_{*}\left(1+\hat{s} \Delta_{b} / r_{*}\right)$ with $\hat{s}$ the magnetic shear, $q_{*}=q\left(r_{*}\right)$, we can write:

$$
\frac{1}{r\left(v_{\|} b^{\theta}+v_{d}^{\theta}\right)}=\frac{q_{*} R}{r_{*} v_{\|}}\left[1+\hat{s} \frac{\Delta_{b}}{r_{*}}+\mathcal{O}\left(\frac{\Delta_{b}^{2}}{r_{*}^{2}}\right)+\mathcal{O}\left(\epsilon^{2}\right)\right] .
$$

Now we easily calculate the integral in Eq. (6). Then, taking $\omega_{b}=v /\left(q R_{0}\right)$, we obtain:

$$
\omega_{D} \equiv-\left\langle q v_{d}^{\theta}\right\rangle=\xi \frac{v^{2}}{R_{0}^{2} \omega_{B 0}}
$$

where

$$
\xi=q\left(\frac{1}{q^{2}}-1-\frac{3 \Delta^{\prime}}{2 \epsilon}-\frac{r \Delta^{\prime \prime}}{2 \epsilon}+\sigma_{v} \frac{\hat{s} q \rho}{r \epsilon}\right),
$$

$\omega_{B 0}=e B_{0} /(M c)$ with $B_{0}=B(r=0)$, and $\Delta^{\prime \prime}=d^{2} \Delta / d r^{2}$.

The derivatives of the Shafranov shift can be expressed in terms of plasma parameters as follows, see, e.g., Ref. ${ }^{7}$ :

$$
\begin{gathered}
\Delta^{\prime}=\epsilon\left(\beta_{\theta}+0.5 l_{i}\right), \\
r \Delta^{\prime \prime}=\epsilon\left[1-(3-2 \hat{s})\left(\beta_{\theta}+0.5 l_{i}\right)\right]+\alpha_{p},
\end{gathered}
$$

where $l_{i}=2 /\left(r^{2} B_{p o l}^{2}\right) \int_{0}^{r} B_{p o l}^{2} r d r$ is the internal inductance, $B_{p o l}$ is the poloidal magnetic field, $\beta_{\theta}=8 \pi(\bar{p}-p) / B_{\text {pol }}^{2}$ with $\bar{p}=\left(2 / r^{2}\right) \int_{0}^{r} p r d r, p$ the plasma pressure, and $\alpha_{p}=-\left(8 \pi p^{\prime} / B_{0}^{2}\right) R_{0} q^{2}$. Using Eqs. (13) and (14), we write $\xi$ as follows: 


$$
\xi=q^{-1}-q\left[1.5+\hat{s}\left(\beta_{\theta}+0.5 l_{i}\right)+\widetilde{\alpha}_{p}\right]+\sigma_{v} \hat{s} q^{2} \frac{\rho R_{0}}{r^{2}}
$$

where $\widetilde{\alpha}_{p}=\alpha_{p} /\left(2 \epsilon q^{2}\right)=-R_{0}^{2} d \hat{\beta} / d r^{2}$ with $\hat{\beta}=8 \pi p / B_{0}^{2}$ is the quantity associated with plasma diamagnetism. The first term in Eq. (15) describes the paramagnetic precession. The last term takes into account the finite orbit width of the particle. It leads to a contribution of the order of $\rho^{2}$ in $\omega_{d}$; nevertheless, it is considerable when $\rho$ is sufficiently large, which can be the case in spherical tori.

Note that Eq. (15) agrees with the corresponding result of Ref. ${ }^{1}$ but contains two extra terms, the first and last ones (when making this comparison, one should allow for the fact that $\omega_{D}$ was

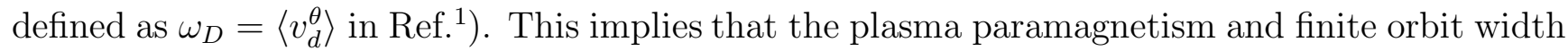
were not taken into account in Ref. ${ }^{1}$.

It follows from Eq. (15) that the magnitude of $\xi$ can considerably vary, depending on plasma parameters. Furthermore, the sign of $\xi$ can change. For instance, $\xi=-0.5$ in a shearless cold plasma with $q=1$, i.e., the precession velocity has the direction opposite to that of the welltrapped particles, $|\xi|$ growing with $q$. Finite plasma pressure and the concomitant diamagnetism strongly enhance the precession when $\beta$ is high, leading to $\widetilde{\alpha}_{p}>1$. The presence of the shear increases $|\xi|$ (but $\xi<0$ ) when $\sigma_{v}<0$ and can decrease it or reverse its sign when $\sigma_{v}>0$. We conclude from this that the largest precession takes place for counter-passing particles in plasmas with high $\beta$ and considerable shear, in which case $|\xi| \gg 1$ even for $q \sim 1$. Note that the maximum $|\xi|$ can be estimated as $q R_{0} / r$ and the maximum $\omega_{D} /\left(q \omega_{b}\right)$, as $q \rho / r$ [this follows from the inequality $\left.\omega_{D} \leq\left.\left(q v_{D}^{\theta}\right)\right|_{\max }\right]$.

One can see that plasma shaping has a considerable influence on the precession. First of all, it reduces $v_{d}$ (for given $q$ ) and the orbit width $\Delta_{b}$ by a factor of $k$, where $k$ is the plasma elongation. Therefore, the last term in Eq. (12) will be decreased by a factor of $k^{2}$. The dependence of other terms on $k$ is more complicated. The effect of plasma shaping on the precession is studied in the next section, where near-axis particles are considered. 


\section{PRECESSION OF CIRCULATING AND POLOIDALLY TRAPPED PARTICLES IN THE NEAR-AXIS REGION}

\section{A. Particles with narrow orbits in non-circular plasmas}

In order to study the precession of the near-axis particles, it is convenient to use the following expansion of the magnetic field strength in a Taylor series (we restrict ourselves to second-order terms):

$$
B=B_{0}\left(1-\epsilon \cos \theta+\alpha \epsilon^{2}+\sigma \epsilon^{2} \cos ^{2} \theta\right)
$$

where $\alpha$ and $\sigma$ are parameters. Equation (16) is valid for plasmas of any shape of the cross-section with up-down symmetry. It is based on the results of Refs. ${ }^{8,9}$ and was used to study the precession in Refs. ${ }^{4,1}$. The parameters $\alpha$ and $\sigma$ were evaluated in Ref. ${ }^{4}$ by adjusting visually the magnetic field strength given by Eq. (16) to that in a numerically calculated high- $\beta$ equilibrium in the NSTX

spherical torus (National Spherical Torus eXperiment ${ }^{10}$ ), the aim being to have agreement in as large region as possible (rather than to have the best agreement in the near-axis region). Such an approach is rather rough; in addition, it does not enable one to determine the dependence of $\alpha$ and $\sigma$ on plasma parameters. Therefore, in Appendix B of this work we calculate $\alpha$ and $\sigma$ in the near-axis region in terms of plasma parameters, the Boozer coordinates being used. The obtained expressions are given below:

$$
\begin{gathered}
\alpha=-\frac{k^{2}}{2 q_{0}^{2}}+\frac{9 k^{2}+2}{6 k^{2}+2}+\frac{3 k^{2}-2}{3 k^{2}+1} \frac{\delta R_{0}}{r}+\left(\frac{1}{2}+\frac{3 q_{0}^{2}}{3 k^{2}+1}\right) \widetilde{\alpha}_{p}, \\
\sigma=\frac{k^{2}-1}{2 q_{0}^{2}}-\frac{k^{2}}{3 k^{2}+1}-\frac{3 k^{2}-1}{3 k^{2}+1} \frac{\delta R_{0}}{r}-\frac{2 q_{0}^{2}}{3 k^{2}+1} \widetilde{\alpha}_{p},
\end{gathered}
$$

where $\delta$ is the flux-surface triangularity (the horizontal shift of the upper and lower tips of the flux surface divided by the flux-surface half-width at the equatorial plane), $\delta R_{0} / r=$ const, $r=$ $\left[2 \psi /\left(k B_{0}\right)\right]^{1 / 2}$ (in main order this coincides with the common definition of $r$ as the half-width of the flux surface at the equatorial plane). Equations (17) and (18) do not depend on the magnetic 
shear and non-parabolic pressure profiles, which implies that terms of higher order (in $\epsilon$ ) should be taken into account when the mentioned factors become important. Note that Eqs. (17) and (18) are obtained in Boozer coordinates; another choice of the poloidal angle $\theta$ may result in a modification of $\alpha$ and $\sigma$.

Equations (16)-(18) determine the magnetic field strength. Knowing $B$, we can use the wellknown expression for the covariant $\psi$-component of the field line curvature [see, e.g., Ref. ${ }^{7}$ and Eq. (A2)], $\kappa_{\psi}=B^{-2}(\partial / \partial \psi)\left(B^{2} / 2+4 \pi p\right)$. Then

$$
v_{d}^{\theta}=\frac{B^{\varphi} b_{\varphi} v^{2}}{k \omega_{B 0} r}\left[\left(-1+\frac{\lambda}{2} \frac{B}{B_{0}}\right) \frac{\partial}{\partial r} \frac{1}{B}+\frac{4 \pi v_{\|}^{2}}{v^{2} B^{3}} \frac{d p}{d r}\right],
$$

where $\lambda=\mu B_{0} / \mathcal{E}$ with $\mathcal{E}$ the particle energy. As in the previous section, we consider circulating narrow-orbit particles, but in a non-circular plasma and with $\mu \neq 0$. Substituting Eq. (19) in Eq. (6), we obtain:

$$
\omega_{D}=\frac{\omega_{b} v^{2}}{k \omega_{B 0}} \oint \frac{d \theta}{2 \pi} \frac{q^{2} B_{\varphi}}{\left|v_{\|}\right| r}\left[\left(1-\frac{\lambda B}{2 B_{0}}\right) \frac{\partial}{\partial r} \frac{1}{B}-\frac{v_{\|}^{2}}{v^{2}} \frac{4 \pi}{|B|^{3}} \frac{d p}{d r}\right] .
$$

We restrict ourselves to well-circulating particles, assuming that $\lambda \ll 1$. Then $v_{\|}=$ $\sigma_{v} v\left[\left(1-0.5 \lambda B / B_{0}\right)+\mathcal{O}\left(\lambda^{2}\right)\right]$. Therefore, Eq. (20) can be written as follows (the terms of the order of $\lambda^{2}$ are neglected):

$$
\omega_{D}=\frac{\omega_{b} v}{k \omega_{B 0}} \oint \frac{d \theta}{2 \pi} \frac{q^{2}}{r} B_{\varphi}\left(\frac{\partial}{\partial r} \frac{1}{B}-\frac{\left|v_{\|}\right|}{v} \frac{4 \pi}{|B|^{3}} \frac{d p}{d r}\right) .
$$

Equations (11), (16), and (21) yield:

$$
\xi=\frac{q_{0}}{k}\left[1-2 \alpha-\sigma+\widetilde{\alpha}_{p}\left(1-\frac{\lambda}{2}\right)\right] \frac{\omega_{b}}{\omega_{b 0}}
$$

where

$$
\frac{\omega_{b}}{\omega_{b 0}} \approx 1-0.5 \lambda\left(1+\alpha \epsilon^{2}+0.5 \sigma \epsilon^{2}\right)
$$

and $\omega_{b 0}=\omega_{b}(\lambda=0)=v /\left(q R_{0}\right)$. Now, putting $\alpha$ and $\sigma$ given by Eqs. (17) and (18) in Eq. (23), we obtain: 


$$
\xi=\frac{q_{0}}{k}\left[\frac{k^{2}+1}{2 q_{0}^{2}}-\frac{5 k^{2}+1}{3 k^{2}+1}-3 \frac{k^{2}-1}{3 k^{2}+1} \frac{\delta R_{0}}{r}-\left(\frac{4 q_{0}^{2}}{3 k^{2}+1}+\frac{\lambda}{2}\right) \widetilde{\alpha}_{p}\right] \frac{\omega_{b}}{\omega_{b 0}} .
$$

Note that in the particular case of a shearless plasma with a circular cross section $(k=1, \delta=0)$ Eq. (24) coincides with Eq. (15) for $q=q_{0}$.

Equation (24) shows that the role of the paramagnetic precession grows with the plasma elongation, whereas the role of the diamagnetic precession drops. Therefore, $\xi$ vanishes at certain $k$ and becomes positive when $k$ is large enough, in which case the paramagnetic precession dominates. For instance, Eq. (24) yields $\xi \approx 0$ for $k=2, q=1, \delta R_{0} / r=0.8, \widetilde{\alpha}_{p}=1$, and $\lambda=0$. On the other hand, the diamagnetic precession overcomes the paramagnetic precession in high- $\beta$ plasmas of spherical tori with moderate $k$ and sufficiently peaked plasma pressure, which leads to $|\xi|>1$ with $\xi<0$. Taking, e.g., $\beta=\beta_{0}\left(1-r^{2} / a^{2}\right)^{2}$ with $a$ the plasma radius, $\beta_{0}=1, R_{0} / a=1.4$, $\delta R_{0} / r=0.8, k=1.5, \lambda=0$, we obtain $\xi=-1.6$.

\section{B. Particles with arbitrary orbit width}

Above we applied the common procedure of calculating the particle precession frequency, which is based on the assumption that the ratio $\Delta r_{b} / r$ is small. Below we will use another procedure, which will enable us to describe also the precession of particles in the near-axis region $(r \lesssim \rho)$, where $\Delta r_{b} \gtrsim r$, i.e., we will consider particles with arbitrary orbit width. The analysis below is relevant to both circulating particles and the semi-trapped particles that have turning points in the poloidal cross section (where $\dot{\theta}=0$ ) and are characterized by small $\lambda$. Such semi-trapped particles do not change the sign of $v_{\|}$in the course of their orbital motion; therefore, as we will see, the frequencies of their motion are described by the same formulae as those of the circulating particles. At the same time, as their orbits do not encircle the magnetic axis, $\langle\dot{\theta}\rangle=0$, which is of importance for their interaction with some electromagnetic perturbations. Note that the analysis below is restricted by the applicability of the approximation of $B$ given by Eqs. (16)-(18).

Considering energetic particles in a steady-state axisymmetric configuration, we neglect the electric field and choose the gauge for the electromagnetic field in the Boozer coordinates as 
follows:

$$
A_{\psi}=0, \quad A_{\theta}=\psi, \quad A_{\varphi}=-\psi_{p}=-\int_{0}^{\psi} \frac{d \psi}{q(\psi)}, \quad \Phi=0
$$

where $\vec{A}$ and $\Phi$ are the vector and scalar potentials of the electromagnetic field, respectively; $\psi_{p}$ is the poloidal magnetic flux.

The action-angle formalism provides a convenient way to calculate the frequencies of the particle motion. ${ }^{11}$ The guiding-center Lagrangian ${ }^{12}$ takes the following form in the action-angle variables (see, e.g., Ref. ${ }^{13}$ for details):

$$
\mathcal{L}=J_{\theta} \dot{\tilde{\theta}}+J_{\varphi} \dot{\tilde{\varphi}}-\mathcal{E}
$$

where $\tilde{\theta}$ and $\tilde{\varphi}$ are specially defined poloidal and toroidal coordinates on the drift surfaces, respectively (canonical angles);

$$
\begin{aligned}
J_{\theta}\left(\mu, \mathcal{E}, J_{\varphi}\right) & =\frac{1}{2 \pi} \oint_{J_{\varphi}=\text { const }, \varphi=\mathrm{const}} d \vec{r} \cdot\left(\frac{e}{c} \vec{A}+M v_{\|} \vec{b}\right) \\
& =\frac{e}{2 \pi c} \oint_{J_{\varphi}=\mathrm{const}}\left[d \theta\left(\psi+\rho_{\|} B_{\theta}\right)+d \psi \rho_{\|} B_{\psi}\right],
\end{aligned}
$$

is the poloidal action; $\rho_{\|}=v_{\|} / \omega_{B} ; J_{\varphi}(\psi, \theta, \mu, \mathcal{E})=(e / c)\left(-\psi_{p}+\rho_{\|} B_{\varphi}\right)$ is the toroidal canonical angular momentum (toroidal action). $J_{\theta}$ and $J_{\varphi}$ are, in fact, the integrals (divided by $2 \pi$ ) of the canonical momentum along arbitrary poloidal and toroidal contours, respectively, lying on the particle drift torus. One can show, in particular, that $J_{\theta}$ reduces to the longitudinal adiabatic invariant, $\oint d s M v_{\|} /(2 \pi)$, for the trapped particles with narrow orbits. In Eq. (26) we consider $\tilde{\theta}, \tilde{\varphi}, \mathcal{E}$ and $J_{\varphi}$ as variables and $J_{\theta}$ as a function of $\mathcal{E}$ and $J_{\varphi}$. As the average frequencies of the toroidal and poloidal motion of a particle cannot depend on the choice of the poloidal and toroidal coordinates, we can obtain them from the Euler-Lagrange equations resulting from Eq. (26):

$$
\omega_{b}=\left(\frac{\partial J_{\theta}}{\partial \mathcal{E}}\right)^{-1}, \quad \frac{\omega_{\varphi}}{\omega_{b}}=-\frac{\partial J_{\theta}}{\partial J_{\varphi}} .
$$

where $\omega_{\varphi}$ is the frequency of the toroidal motion, $\omega_{b}$ is the bounce/transit frequency. Note that Eq. (28) is exact for given actions, and Eq. (27) is exact in the framework of the first-order Lagrangian (or Hamiltonian) guiding-center theory; therefore, hitherto we have used no assumptions on the orbit width, the aspect ratio etc. 
Thus, we need to calculate $J_{\theta}$ as a function of $J_{\varphi}, \mathcal{E}$, and $\mu$. With this aim, we assume that $\rho / R_{0} \sim r / a \sim r / R_{0} \sim \epsilon \ll 1, r^{2} d \hat{\beta} / d\left(r^{2}\right) \sim \epsilon^{2}$ (this implies, in particular, that $\left.\widetilde{\alpha}_{p} \sim \alpha \sim \sigma \sim 1\right)$. This ordering is relevant, in particular, to near-axis particles in a spherical torus with moderately high $\beta$. In addition, we assume that $\lambda \epsilon \ll 1-\lambda$, which means that the particles are well circulating in the sense that $v_{\|}$is not close to zero anywhere on the particle orbits. Nevertheless, we do not demand that the orbits enclose the magnetic axis, allowing for poloidally trapped particles.

Using Eq. (16), we obtain

$$
\rho_{\|}=\rho_{\| 0}\left[1+\Lambda\left(x-\alpha x^{2}-\alpha y^{2}-\sigma x^{2}\right)+\frac{8-12 \lambda+3 \lambda^{2}}{8(1-\lambda)^{2}} x^{2}+\mathcal{O}\left(\epsilon^{3}\right)\right],
$$

where $\rho_{\| 0}=\rho_{\|}(r=0)=(1-\lambda)^{1 / 2} \sigma_{v} v / \omega_{B 0}, \Lambda=(1-\lambda / 2) /(1-\lambda), x=\left(r / R_{0}\right) \cos \theta, y=$ $\left(r / R_{0}\right) \sin \theta$. It follows from Eq. (B11) that $\psi_{p}=k B_{0} r^{2} /\left(2 q_{0}\right)\left[1+\mathcal{O}\left(\epsilon^{2}\right)\right]$. Then, using Eq. (B12), we can write the following asymptotic expression for $J_{\varphi}$ :

$$
\begin{aligned}
J_{\varphi} & =\frac{e B_{0} R_{0}^{2}}{c}\left[\frac{\rho_{\|}}{R_{0}}+\Lambda \frac{\rho_{\|}}{R_{0}} x-A_{x} x^{2}-A_{y} y^{2}+\mathcal{O}\left(\epsilon^{4}\right)\right] \\
& =-\frac{e B_{0} R_{0}^{2}}{c}\left[A_{x} \xi^{2}+A_{y} y^{2}-C+\mathcal{O}\left(\epsilon^{4}\right)\right]
\end{aligned}
$$

where $\xi=x-d, d=\Lambda \rho_{\| 0} /\left(2 A_{x} R_{0}\right)$,

$$
\begin{gathered}
A_{x}=A_{y}+\frac{\rho_{\|}}{R_{0}}\left[\Lambda \sigma-\frac{8-12 \lambda+3 \lambda^{2}}{8(1-\lambda)^{2}}\right], \\
A_{y}=\frac{k}{2 q_{0}}+\frac{\rho_{\|}}{R_{0}}\left(\Lambda \alpha-\frac{k}{2} R_{0} B_{\varphi(2)}\right),
\end{gathered}
$$

and $C=\rho_{\|} / R_{0}+\rho_{\|}^{2} \Lambda^{2} /\left(4 A_{x} R_{0}^{2}\right)$. Thus, in the coordinates $(\xi, y)$ the particle orbits are ellipses with the center at the origin (to the considered order).

Equation (27) takes the following form in these coordinates:

$$
\begin{aligned}
J_{\theta} & =\sigma_{v} \frac{e B_{0} R_{0}^{2}}{2 \pi c} \int_{S} d \xi d y\left[k+2 \frac{\rho_{\|} B_{\theta}}{r^{2} B_{0}}+(\xi+d) \frac{B_{\theta}}{r^{2} B_{0}} \frac{\partial \rho_{\|}}{\partial \xi}+y \frac{B_{\theta}}{r^{2} B_{0}} \frac{\partial \rho_{\|}}{\partial y}\right. \\
& \left.+k y \frac{\partial}{\partial \xi}\left(\rho_{\|} B_{\psi}\right)-k(\xi+d) \frac{\partial}{\partial y}\left(\rho_{\|} B_{\psi}\right)+\mathcal{O}\left(\epsilon^{2}\right)\right]
\end{aligned}
$$


where $S\left(J_{\varphi}, \mu, \mathcal{E}\right)$ is the interior of the contour $J_{\varphi}=$ const on the $(\xi, y)$ plane. Note that $\sigma_{v}$ has arisen in Eq. (33) because the integration in Eq. (33) is performed in the clockwise direction for the counter-circulating particles and in the counterclockwise direction for the co-circulating ones. Taking into account Eqs. (29), (B22), and (B27), we find that all non-constant terms are negligible:

$$
J_{\theta}=\sigma_{v} \frac{e B_{0} R_{0}^{2}}{2 \pi c} \int_{S} d \xi d y\left[k+\left(k^{2}+1\right) \frac{\rho_{\| 0}}{q_{0} R_{0}}+\mathcal{O}\left(\epsilon^{2}\right)\right]
$$

Recalling that $S$ is the interior of the ellipse described by Eq. (30), we obtain:

$$
J_{\theta}=\frac{\sigma_{v}}{2}\left[k+\left(k^{2}+1\right) \frac{\rho_{\| 0}}{q_{0} R_{0}}\right]\left(A_{x} A_{y}\right)^{-1 / 2}\left(\frac{e B_{0} R_{0}^{2}}{2 \pi c} C-J_{\varphi}\right)+\mathcal{O}\left(\epsilon^{4}\right) .
$$

Finally, substituting Eq. (35) for $J_{\theta}$ and Eq. (B20) for $B_{\varphi(2)}$ in Eq. (28) and taking into account that $C-2 \pi c J_{\varphi} /\left(e B_{0} R_{0}^{2}\right) \sim \epsilon^{2}$ (because this quantity is the square of the ellipse $S$ of the size $\sim \epsilon$ ), we obtain

$$
\begin{gathered}
\frac{\omega_{\varphi}}{\omega_{b}}=\sigma_{v} q_{0}\left\{1-\frac{q_{0} \rho_{\| 0}}{k R_{0}}\left[(2 \alpha+\sigma) \Lambda-\frac{1-\frac{3}{2} \lambda+\frac{3}{8} \lambda^{2}}{(1-\lambda)^{2}}-\widetilde{\alpha}_{p}\right]+\mathcal{O}\left(\epsilon^{2}\right)\right\}, \\
\omega_{b}=\frac{\left|v_{\| 0}\right|}{q_{0} R_{0}}\left\{1+\frac{q_{0} \rho_{\| 0}}{k R_{0}}\left[(2 \alpha+\sigma) \Lambda-\frac{2-\frac{7}{2} \lambda+\frac{9}{8} \lambda^{2}}{(1-\lambda)^{2}}-\widetilde{\alpha}_{p}\right]+\mathcal{O}\left(\epsilon^{2}\right)\right\}, \\
\omega_{\varphi}=\frac{v_{\| 0}}{R_{0}}\left[1-\frac{\rho_{\| 0} q_{0}}{k R_{0}} \frac{1-2 \lambda+\frac{3}{4} \lambda^{2}}{(1-\lambda)^{2}}+\mathcal{O}\left(\epsilon^{2}\right)\right],
\end{gathered}
$$

where $v_{\| 0}=v_{\|}(r=0)=(1-\lambda)^{1 / 2} \sigma_{v} v$. We observe that the frequencies given by Eqs. (36)-(38) do not depend on $J_{\varphi}$ and, therefore, are the same for conventional circulating and poloidally trapped particles. For the circulating particles, these obtained results agree with those obtained above for the narrow-orbit case. Indeed, expanding the $\rho_{\|}$term of Eq. (36) in degrees of $\lambda$ for $\lambda \ll 1$, we see that the two approaches give the same expressions for the precession [see Eq. (22)].

For the semi-trapped particles, we can interpret the toroidal motion described by Eq. (38) in two ways. First, one can show that $\omega_{\varphi}$ equals to $v_{\|} / R$ at the point $x=d, y=0$, which is the center of the orbits and the location of the stagnation orbit for given $\mathcal{E}$ and $\mu$. Hence, like for 
circulating particles, the toroidal frequency can be interpreted as resulting from the longitudinal motion. Second, we recall that the particles are trapped in the poloidal direction and, in fact, do not follow the field lines. The well-known formula for the toroidal precession frequency of the trapped particles gives $\omega_{\varphi}=q v_{d}^{\theta}$ for the particles in the vicinity of the stagnation orbit (when $\cos \theta \approx 1)$. As for such particles the vertical velocities due to the longitudinal and drift motion compensate for each other, $v_{d}^{\theta}=v_{\|} /\left(q R_{0}\right)$, we recover the main term of Eq. (38). Thus, the main term of Eq. (38) can also be considered as a result of the toroidal precession of semi-trapped particles.

\section{SUMMARY AND CONCLUSIONS}

We have considered the toroidal precession by using both the conventional approach applicable to particles with $\Delta_{b} \ll r$ and the approach based on the action integrals and quasi-cartesian coordinates. The latter enabled us to consider near-axis well-circulating particles and semi-trapped particles [i.e., the particles that do not encircle the magnetic axis but do not change $\operatorname{sgn}\left(v_{\|}\right)$ during their motion] with small $\lambda=\mu B_{0} / \mathcal{E}$, which is especially important for the energetic ions with the large Larmor radius (e.g., in spherical tori). In addition, an analysis of equilibrium for a plasma with a non-circular cross section is carried out in the paraxial approximation. Due to this analysis, coefficients in a Taylor expansion of the magnetic field strength are found in terms of plasma characteristics. An expression for the radial component of the magnetic field line curvature $\left(\kappa_{\psi}\right)$ is derived, taking into account the poloidal magnetic field, which is responsible for the paramagnetic precession. Finally, we obtained expressions for the precession frequency, which are convenient for the practical use.

A general conclusion which follows from our work is that the precession of the toroidally passing particles can strongly vary $\left(0 \leq|\xi| \lesssim R_{0} / r\right.$, and $\xi$ can be either positive or negative), depending on several factors. These factors are the plasma shaping and $\beta$, the aspect ratio $R_{0} / r$, and the safety factor profile. Because of this, the effects of the precession of toroidally passing energetic 
ions on plasma instabilities (the fishbone mode, sawtooth oscillations etc.) and the transport of the energetic ions in MHD-active plasmas are rather sensitive to specific experimental conditions.

\section{ACKNOWLEDGMENTS}

The authors thank V. S. Marchenko for a discussion of the work. The research described in this publication was made possible in part by the Award No. UP2-2419-KV-02 of the U.S. Civilian Research \& Development Foundation and the Government of Ukraine and the U.S. Department of Energy under contract number DE-AC02-76-CHO3073.

\section{APPENDIX A: FIELD LINE CIRVATURE IN A CIRCULAR TOKAMAK}

In this Appendix we calculate the field line curvature in an axisymmetric toroidal magnetic configuration with a circular cross section. We assume that $\epsilon \equiv r / R_{0} \ll 1$ and $\Delta \hat{\beta} \equiv 8 \pi \Delta p / B_{0}^{2} \sim$ $\epsilon^{2}$, where $\Delta p$ is the pressure variation in the considered region, which means that we consider either a large-aspect-ratio tokamak or the central part of a spherical torus with moderately high central $\beta$.

It follows from the force balance equation,

$$
(\nabla \times \vec{B}) \times \vec{B}=4 \pi \nabla p
$$

that the field line curvature, $\vec{\kappa}=(\vec{b} \cdot \nabla) \vec{b}$, can be found as follows:

$$
\vec{\kappa}=\frac{1}{2 B^{2}}\left[\nabla\left(B^{2}+8 \pi p\right)-\vec{b} \nabla_{\|} B^{2}\right]
$$

As $\nabla_{\|} B \sim \epsilon \nabla_{\perp} B$, the second term in Eq. (A2) is $\epsilon^{2}$ times smaller than the first one and will be neglected.

An axisymmetric magnetic field can be presented in the form

$$
\vec{B}=F(r) \vec{e}^{\varphi}+\frac{1}{\sqrt{g}} \vec{e}_{\theta} \frac{d \psi_{p}}{d r},
$$


where the coordinate system $(r, \theta, \varphi)$ is taken so that the radial coordinate is constant on each flux surface; $F(r)=B_{0} R_{0}\left[1+\mathcal{O}\left(\epsilon^{2}\right)\right]$ is a function of $r$ describing the plasma diamagnetism; $\vec{e}_{j}$ and $\vec{e}^{j}, j=r, \theta, \varphi$, are the corresponding co- and contravariant base vectors; $g$ is the determinant of the metric tensor. Note that we do not assume in this Appendix that we use Boozer coordinates. One can show that in a circular tokamak to main order in $\epsilon$

$$
g_{\theta \theta}=r^{2}, \quad \sqrt{g}=r R_{0}, \quad g_{r \theta} \sim \epsilon r
$$

where $g_{r \theta}$ and $g_{\theta \theta}$ are the corresponding components of the covariant metric tensor (see, e.g., Ref. $\left.{ }^{14}\right)$. Then the poloidal component of the magnetic field, $B_{\text {pol }}$, in a circular tokamak [which is described by the second term of Eq. (A3)] is constant over a flux surface to main order,

$$
B_{p o l}=\left(\frac{g_{\theta \theta}}{g}\right)^{1 / 2} \frac{d \Psi}{d r}=\frac{\epsilon}{q} B_{0}[1+\mathcal{O}(\epsilon)]
$$

From Eqs. (A2), (A3), and (A5), the curvature is

$$
\vec{\kappa}=\frac{1}{2 B^{2}} \nabla\left(\frac{F^{2}}{R^{2}}+\frac{\epsilon^{2} B_{0}^{2}}{q^{2}}+8 \pi p\right)+\mathcal{O}\left(\epsilon^{2} R_{0}^{-1}\right) .
$$

Using Eqs. (A3)-(A5), we can write Eq. (A1) as follows:

$$
8 \pi \frac{d p}{d r}+\frac{1}{R^{2}} \frac{d\left(F^{2}\right)}{d r}+\frac{d}{d r} \frac{\epsilon^{2} B_{0}^{2}}{q^{2}}=-\frac{2 \epsilon^{2}}{r q^{2}} B_{0}^{2}+\mathcal{O}\left(\epsilon^{3} \frac{B_{0}^{2}}{r}\right) .
$$

Comparing Eqs. (A6) and (A7), we find

$$
\vec{\kappa}=-\frac{1}{R} \nabla R-\frac{\epsilon^{2}}{r q^{2}} \nabla r+\mathcal{O}\left(\epsilon^{2} R_{0}^{-1}\right) .
$$

Equation (7) immediately follows from Eq. (A8). The first and second terms of Eq. (A8) can be interpreted as the contributions of the toroidal and poloidal curvature of the field lines, respectively. In particular, we recover from Eq. (A8) the following formula for the scalar curvature: ${ }^{15}$

$$
\kappa=\frac{1}{R}\left(1+\frac{2 \epsilon}{q^{2}} \nabla R \cdot \nabla r\right)^{1 / 2}=R_{0}^{-1}\left[1+\epsilon\left(q^{-2}-1\right) \cos \theta\right]
$$

To find $\kappa_{r}$ from Eq. (A8) with the accuracy $\sim \epsilon$, which is required for calculating the precession, we need to determine $\partial R / \partial r$ with the same accuracy. It turns out that $\partial R / \partial r$ at this order depends 
on the choice of the poloidal angle. Taking into account that non-circularity effects appear only in the second order in a circular tokamak, we present the flux surfaces as follows: ${ }^{14}$

$$
\begin{gathered}
R=R_{0}-\Delta(r)+r \cos \vartheta+\mathcal{O}\left(r \epsilon^{2}\right), \\
z=r \sin \vartheta+\mathcal{O}\left(r \epsilon^{2}\right), \\
\vartheta=\theta+\gamma(r) \sin \theta+\mathcal{O}\left(\epsilon^{2}\right),
\end{gathered}
$$

where $\Delta \sim \epsilon r, \vartheta$ is the "geometrical" poloidal angle, $\theta$ is the poloidal angle of the chosen coordinate system, $\gamma \sim \epsilon$ is the angle correction providing the desired properties of the coordinates. It follows from Eqs. (A10)-(A12) that the metric tensor determinant of the coordinates $(r, \theta, \varphi)$ is given by

$$
\sqrt{g}=R_{0} r\left[1+\left(\epsilon+\gamma-\Delta^{\prime}\right) \cos \theta+\mathcal{O}\left(\epsilon^{2}\right)\right] .
$$

Equation (B2) shows that in Boozer coordinates the product $B^{2} \sqrt{g}$ does not depend on $\theta$. Then, using Eqs. (16) and (A13), we obtain

$$
\gamma=\epsilon+\Delta^{\prime}
$$

Equation (8) follows from Eqs. (A10), (A12), and (A14).

Another widely used coordinate system is the system of coordinates in which the toroidal angle $\phi$ is the "geometrical" angle and the poloidal angle is chosen so that the field lines are "straight", i.e., the ratio $B^{\phi} / B^{\theta}=q(r)$ does not depend on $\theta$. In such coordinates $B^{\phi}=B_{\phi} / R^{2}$, and it follows from Eq. (A3) that $\sqrt{g} / R^{2}$ is independent on $\theta$. To required order, this condition also results in Eq. (A14), and $R$ in such coordinates is also given by Eq. (8). In fact, as one can see from Eq. (B21), the difference between the Boozer toroidal angle and the "geometrical" one is very small, $\sim \epsilon^{3}$, when $k=1$.

The Meiss-Hazeltine flux coordinates ${ }^{6}$ are defined by the condition that the radial covariant component of $\vec{B}$ vanishes. To considered order, they coincide with the "geometrical" flux coordinates in a circular tokamak. Therefore, Eq. (8) holds in these coordinates, too. The fact that the 
difference between the Boozer and Meiss-Hazeltine angles is small, $\sim \epsilon^{3}$ is not surprising since $B_{\psi}$ in the Boozer coordinates is rather small, see Eq. (B27). Moreover, unlike the similar estimate of the difference between the "geometrical" and Boozer coordinates, this estimate holds also in the case of $k \neq 1$.

\section{APPENDIX B: AXISYMMETRIC EQUILIBRIUM IN THE PARAXIAL APPROXIMATION}

The aim of the calculations presented in this Appendix is to obtain the parameters of a general plasma equilibrium with axial and up-down symmetries in the vicinity of the magnetic axis. The derivation employs a Taylor expansion of the plasma equilibrium in the radial coordinate, the ratio of the flux-surface radius to the other characteristic lengths being used as a small parameter. An advantage of this approach is its simplicity; that is why it was used for general three-dimensional configurations. ${ }^{8,16,17}$ However, its serious drawback is that one has to get to high order in order to study any effects of the magnetic shear, non-parabolic pressure profiles etc. We use a modification of this approach for the Boozer flux coordinates, which was suggested in Ref. ${ }^{18}$ (see also Ref. ${ }^{19}$ ).

The contravariant and covariant representations of the magnetic field in Boozer coordinates have the form ${ }^{5}$

$$
\begin{aligned}
\vec{B} & =\frac{1}{\sqrt{g}} \vec{e}_{\varphi}+\frac{\iota(\psi)}{\sqrt{g}} \vec{e}_{\theta} \\
& =B_{\psi}(\psi, \theta) \nabla \psi+B_{\theta}(\psi) \nabla \theta+B_{\varphi}(\psi) \nabla \varphi
\end{aligned}
$$

where $\vec{e}_{\theta} \equiv \partial \vec{r} / \partial \theta$ and $\vec{e}_{\varphi} \equiv \partial \vec{r} / \partial \varphi$ are covariant base vectors, $g$ is the determinant of the metric tensor, $\iota=q^{-1}$. It follows from Eq. (B1) that

$$
\sqrt{g}=B^{-2}\left(B_{\varphi}+\iota B_{\theta}\right)
$$

Following Ref. ${ }^{18}$, we write the spatial position of a point, $\vec{r}$, as a function of the flux coordinates $(\psi, \theta, \varphi):$ 


$$
\vec{r}(\psi, \theta, \varphi)=\vec{r}_{0}(\varphi)+X(\psi, \theta) \hat{\vec{k}}(\varphi)+Y(\psi, \theta) \hat{\vec{b}}(\varphi)+Z(\psi, \theta) \hat{\vec{\tau}}(\varphi)
$$

where $\vec{r}_{0}(\varphi)$ is the spatial position of the magnetic axis; $\hat{\vec{\tau}}(\varphi), \hat{\vec{k}}(\varphi)$, and $\hat{\vec{b}}(\varphi)$ are the tangent, normal, and binormal (the Frenet unit vectors) of the magnetic axis, respectively. The unknown functions $X(\psi, \theta), Y(\psi, \theta)$, and $Z(\psi, \theta)$ are assumed to satisfy the condition $X(\psi=0)=Y(\psi=$ $0)=Z(\psi=0)=0$. Then they are presented as power series in $\varepsilon \equiv \psi^{1 / 2}$ :

$$
X(\psi, \theta)=\varepsilon X_{(1)}(\theta)+\varepsilon^{2} X_{(2)}(\theta)+\varepsilon^{3} X_{(3)}(\theta)+\ldots,
$$

and the same for $Y(\psi, \theta)$ and $Z(\psi, \theta)$. If the functions $\psi(\vec{x})$ and $\varphi(\vec{x})$ are analytic, as well as $\theta(\vec{x})$ aside from $\psi=0$, the Fourier series of $X_{(1)}(\theta), Y_{(1)}(\theta)$, and $Z_{(1)}(\theta)$ in $\theta$ can contain only first harmonics,

$$
X_{(1)}(\theta)=X_{(1) s} \sin \theta+X_{(1) c} \cos \theta ;
$$

those of $X_{(2)}(\theta), Y_{(2)}(\theta)$, and $Z_{(2)}(\theta)$, zeroth and second harmonics; etc. ${ }^{18}$

Expressions for the covariant base vectors $\vec{e}_{\psi} \equiv \partial \vec{r} / \partial \psi, \vec{e}_{\theta}$, and $\vec{e}_{\varphi}$ can be obtained by differentiating Eq. (B3):

$$
\begin{gathered}
\vec{e}_{\psi}=\frac{1}{2 \varepsilon}\left(\frac{\partial X}{\partial \varepsilon} \hat{\vec{\kappa}}+\frac{\partial Y}{\partial \varepsilon} \hat{\vec{b}}+\frac{\partial Z}{\partial \varepsilon} \hat{\vec{\tau}}\right), \\
\vec{e}_{\theta}=\frac{\partial X}{\partial \theta} \hat{\vec{\kappa}}+\frac{\partial Y}{\partial \theta} \hat{\vec{b}}+\frac{\partial Z}{\partial \theta} \hat{\vec{\tau}}, \\
\vec{e}_{\varphi}=\frac{Z}{R_{0}} \frac{d l}{d \varphi} \hat{\vec{\kappa}}+\left(\frac{d l}{d \varphi}-\frac{X}{R_{0}} \frac{d l}{d \varphi}\right) \hat{\vec{\tau}},
\end{gathered}
$$

where $l(\varphi)$ is the distance along the magnetic axis, $d l / d \varphi=\left|d \vec{r}_{0} / d \varphi\right|$, and the Frenet formulae ${ }^{20}$ have been used to find the $\varphi$ derivatives of the Frenet unit vectors.

It is also assumed that ${ }^{18}$

$$
\begin{gathered}
B(\psi, \theta)=B_{(0)}+B_{(1)}(\theta) \varepsilon+B_{(2)}(\theta) \varepsilon^{2}+\ldots, \\
B_{\psi}(\psi, \theta)=B_{\psi(0)}+B_{\psi(1)}(\theta) \varepsilon+B_{\psi(2)}(\theta) \varepsilon^{2}+\ldots,
\end{gathered}
$$




$$
\begin{gathered}
\iota(\psi)=\iota_{(0)}+\iota_{(2)} \varepsilon^{2}+\iota_{(4)} \varepsilon^{4}+\ldots \\
B_{\varphi}(\psi)=B_{\varphi(0)}+B_{\varphi(2)} \varepsilon^{2}+B_{\varphi(4)} \varepsilon^{4}+\ldots \\
B_{\theta}(\psi)=B_{\theta(2)} \varepsilon^{2}+B_{\theta(4)} \varepsilon^{4}+\ldots
\end{gathered}
$$

with the Fourier spectra of $\left(B^{-2}\right)_{(i)}(\theta)$ and $B_{\psi(i)}(\theta)$ obeying the same rules as those of $X_{(i)}(\theta)$. Using relationships between the Fourier harmonics of $B$ and $B_{\psi},{ }^{18}$ one can show that $B_{\psi(0)}=0$ in axisymmetric configurations.

To find the parameters of the equilibrium, we put Eqs. (B6)-(B8) in the equations

$$
\vec{\Xi} \equiv \iota \vec{e}_{\theta}+\vec{e}_{\varphi}-B_{\psi} \vec{e}_{\theta} \times \vec{e}_{\varphi}-B_{\theta} \vec{e}_{\varphi} \times \vec{e}_{\psi}-B_{\varphi} \vec{e}_{\psi} \times \vec{e}_{\theta}=0
$$

and

$$
B^{-2}(\psi, \theta, \varphi)=\frac{\left|\vec{e}_{\varphi}+\iota \vec{e}_{\theta}\right|^{2}}{\left(B_{\varphi}+\iota B_{\theta}\right)^{2}}
$$

which follow from Eqs. (B1) and (B2) and the well known relationships between the co- and contravariant base vectors: $\nabla \psi=\vec{e}_{\theta} \times \vec{e}_{\varphi} / \sqrt{g}$ etc. Then we will expand the resulting equations in powers of $\varepsilon$ and analyze them order by order. The same will be done with the force balance equation [Eq. (A1)], which takes the following form in the Boozer coordinates:

$$
\frac{d p}{d \psi}=\frac{1}{4 \pi \sqrt{g}}\left(\iota \frac{\partial B_{\psi}}{\partial \theta}-\frac{d B_{\varphi}}{d \psi}-\iota \frac{d B_{\theta}}{d \psi}\right) .
$$

The $\varepsilon^{0}$ order of Eq. (B15) yields:

$$
\frac{d l}{d \varphi}=\frac{B_{\varphi(0)}}{B_{(0)}}
$$

hence, $B_{\varphi(0)}=B_{(0)} R_{0}$. Using Eq. (B17), we present the equation $\hat{\vec{\tau}} \cdot \vec{\Xi}_{(0)}=0$ in the form

$$
X_{(1)} \frac{\partial Y_{(1)}}{\partial \theta}-Y_{(1)} \frac{X_{(1)}}{\partial \theta}=\frac{2}{B_{(0)}} .
$$


Having in mind Eq. (B18), the assumed dependence of $X_{(1)}$ and $Y_{(1)}$ on $\theta$ [see Eq. (B5)], and the up-down symmetry of the configuration, we conclude that they can be put without loss of generality in the following form:

$$
X_{(1)}=\left(\frac{2}{k B_{(0)}}\right)^{1 / 2} \cos \theta, \quad Y_{(1)}=\left(\frac{2 k}{B_{(0)}}\right)^{1 / 2} \sin \theta,
$$

where $k$ is the elongation (ellipticity) of the flux surfaces. It follows from the equation $\left(X_{(1)} \hat{\vec{k}}+\right.$ $\left.Y_{(1)} \hat{\vec{b}}\right) \cdot \vec{\Xi}_{(0)}=0$ that $Z_{(1)}=0$ and, thus, $Z=\mathcal{O}\left(\varepsilon^{2}\right)$. Finally, Eq. (B16) taken in zero order results in the following relationship:

$$
B_{\varphi(2)}=-\frac{B_{\varphi(0)}}{2} \beta_{(2)}-\iota_{(0)} B_{\theta(2)},
$$

where $\beta_{(2)}=8 \pi p_{(2)} / B_{(0)}^{2}$.

Then we proceed to the first-order equations. The equation $\left(X_{(1)} \hat{\vec{k}}+Y_{(1)} \hat{\vec{b}}\right) \cdot \vec{\Xi}_{(1)}=0$ yields:

$$
Z_{(2)}=-\frac{k-k^{-1}}{2 B_{\varphi(0)}} \iota(0) \sin (2 \theta)
$$

Substituting $Z_{(2)}, X_{(1)}$, and $Y_{(1)}$ given by Eqs. (B19) and (B21) in the equation $\left(Y_{(1)} \hat{\vec{k}}-X_{(1)} \hat{\vec{b}}\right) \cdot \vec{\Xi}_{(1)}=$ 0 , we arrive after some algebra at the following relationship between the toroidal current $\left(B_{\theta}\right)$ and $\iota$ near the magnetic axis:

$$
B_{\theta(2)}=\frac{k+k^{-1}}{R_{0}} \iota(0)
$$

The remaining first-order component of Eq. (B14), $\hat{\vec{\tau}} \cdot \vec{\Xi}_{(1)}=0$, yields:

$$
\frac{\partial X_{(1)}}{\partial \theta} Y_{(2)}-\frac{\partial Y_{(1)}}{\partial \theta} X_{(2)}+\frac{Y_{(1)}}{2} \frac{\partial X_{(2)}}{\partial \theta}-\frac{X_{(1)}}{2} \frac{\partial Y_{(2)}}{\partial \theta}=\frac{X_{(1)}}{B_{(0)} R_{0}} .
$$

To proceed further, we need to specify the dependence of $X_{(2)}$ and $Y_{(2)}$ on $\theta$. Taking account of the up-down symmetry and the assumptions made above on the harmonic composition of $X_{(i)}$ and $Y_{(i)}$, we take $X_{(2)}$ and $Y_{(2)}$ in the form

$$
X_{(2)}=X_{(2) 0}+X_{(2) c} \cos (2 \theta), \quad Y_{(2)}=Y_{(2) s} \sin (2 \theta) .
$$

Substituting Eqs. (B19) and (B19) into Eq. (B23), we obtain 


$$
Y_{(2) s}+k X_{(2) c}+k X_{(2) 0}+R_{0}^{-1} B_{(0)}^{-1}=0
$$

The first order of Eq. (B15) gives us the first-order correction to the magnetic field strength:

$$
B_{(1)}=B_{(0)} \frac{X_{(1)}}{R_{0}} .
$$

Expanding Eq. (B16) in powers of $\varepsilon$, we obtain in first order:

$$
B_{\psi(1)}=-\left(\frac{2 B_{(0)}}{k}\right)^{1 / 2} \frac{\beta_{(2)}}{\iota_{(0)}} \sin \theta .
$$

Proceeding to the second order, we put the obtained expressions for $X_{(1,2)}, Y_{(1,2)}, Z_{(2)}, B_{\varphi(0)}$, $B_{\theta(2)}$, and $B_{\psi(1)}$ in the equation $\left(X_{(1)} \hat{\vec{k}}+Y_{(1)} \hat{\vec{b}}\right) \cdot \vec{\Xi}_{(2)}=0$ to find:

$$
\begin{aligned}
Z_{(3)} & =\left(\frac{2}{k B_{(0)}}\right)^{1 / 2} \frac{\iota_{(0)}}{6 R_{0}}\left\{\left[\frac{k-k^{-1}}{2 B_{(0)} R_{0}}+\left(k^{2}-1\right) X_{(2) c}+\left(k^{2}+3\right) X_{(2) 0}\right.\right. \\
& \left.+\left(k-k^{-1}\right) Y_{(2) s}-\frac{4 \beta_{(2)} R_{0}}{\iota_{(0)}^{2}}\right] \sin \theta \\
& \left.+\left[\frac{k-k^{-1}}{2 B_{(0)} R_{0}}+\left(k^{2}+3\right) X_{(2) c}+\left(k^{2}-1\right) X_{(2) 0}-\left(3 k+k^{-1}\right) Y_{(2) s}\right] \sin (3 \theta)\right\} .
\end{aligned}
$$

Performing the same substitutions [and substituting Eq. (B28) for $Z_{(3)}$ as well] in the equation $\left(Y_{(1)} \hat{\vec{k}}-X_{(1)} \hat{\vec{b}}\right) \cdot \vec{\Xi}_{(2)}=0$, we observe that both $\sin (\theta)$ and $\sin (3 \theta)$ harmonics of the resulting equation do not yield independent equations: They are both reduced to the equation

$$
\left(k^{2}-1\right) Y_{(2) s}-2 k\left(k^{2}-1\right) X_{(2) c}-2 k^{3} X_{(2) 0}-k \frac{\beta_{(2)} R_{0}}{\iota_{(0)}^{2}}+\frac{2 k^{2}+1}{R_{0} B_{(0)}}=0 .
$$

Thus, we have obtained only two independent equations, Eqs. (B25) and (B29), for the three parameters describing the flux surface shape in second order, $X_{(2) 0}, X_{(2) c}$, and $Y_{(2) s}$. Analyzing the shape distortions due to these parameters, one can see that they are responsible for the Shafranov shift and triangularity of the flux surfaces, as well as for the poloidal angle correction that provides that the coordinates are indeed Boozer ones. Therefore, one could expect that the equations for these parameters are underdetermined, otherwise the triangularity of the configuration would be predetermined, which is counterintuitive. Let us determine the parameter combinations responsible for the Shafranov shift and triangularity explicitly. Applying the transformation $\theta \rightarrow \theta+\varepsilon \varsigma \sin \theta$ to the parametric equations of the flux surfaces, 


$$
\begin{gathered}
X=\varepsilon\left(\frac{2}{k B_{(0)}}\right)^{1 / 2} \cos \theta+\varepsilon^{2}\left[X_{(2) 0}+X_{(2) c} \cos (2 \theta)\right] \\
Y=\varepsilon\left(\frac{2 k}{B_{(0)}}\right)^{1 / 2} \sin \theta+\varepsilon^{2} Y_{(2) s} \sin (2 \theta)
\end{gathered}
$$

we see that the parameters under consideration are transformed as follows:

$$
X_{(2) 0}=X_{(2) 0}-\tilde{\varsigma}, \quad X_{(2) c}=X_{(2) c}+\tilde{\varsigma}, \quad Y_{(2) s}=Y_{(2) s}+k \tilde{\varsigma}
$$

where $\tilde{\varsigma}=\varsigma /\left(2 k B_{(0)}\right)^{1 / 2}$. As the flux surface shape is invariant to this transformation, the expressions for the Shafranov shift and triangularity must be invariant, too. The triangularity cannot depend on $X_{(2) 0}$ since the variation of this parameter just shifts the flux surfaces without changing their shape. Hence, the only possible expression for the triangularity (up to a constant factor) is $d=Y_{(2) s} / k-X_{(2) c}$. Similarly, as $Y_{(2) s}$ does not affect the flux surface at the equatorial plane, the only reasonable combination for the Shafranov shift is $D=X_{(2) 0}+X_{(2) c}$, which does not include $Y_{(2) s}$ and is invariant to the transformation given by Eq. (B32). One can easily find relationship between the parameters $D$ and $d$ and the conventional Shafranov shift, $\Delta$, and triangularity, $\delta$. We define the radial coordinate, $r$, on a flux surface as the half-width of the surface at the equatorial plane. Then in main order the relation between $r$ and $\psi$ is given by $\psi=k B_{(0)} r^{2} / 2$, and we observe from Eq. (B30) that the Shafranov shift, i.e., the shift of the flux surfaces in the curvature direction at the equatorial plane is given by $\Delta=k B_{(0)} r^{2} D$. To express triangularity in terms of $d$, it is convenient to set $Y_{(2) s}=0$ use the transformation given by Eq. (B32). Then the upper and lower tips of a flux surface correspond to $\theta= \pm \pi / 2$, and we see from Eq. (B30) that the triangularity defined as the shift of the tips in the curvature direction divided by the radius is $\delta=k B_{(0)} r d$.

Solving Eqs. (B25) and (B29) together with the definition of $d$, we obtain

$$
\begin{gathered}
X_{(2) 0}=\frac{1}{3 k^{2}+1}\left[3\left(k^{2}-1\right) d+\frac{5 k^{2}+1}{k B_{(0)} R_{0}}-\frac{4 \beta_{(2)} R_{0}}{\iota_{(0)}^{2}}\right], \\
X_{(2) c}=\frac{1}{3 k^{2}+1}\left[-\left(3 k^{2}-1\right) d-\frac{4 k^{2}+1}{k B_{(0)} R_{0}}+\frac{2 \beta_{(2)} R_{0}}{\iota_{(0)}^{2}}\right],
\end{gathered}
$$


from which we find the Shafranov shift near the magnetic axis:

$$
\Delta=\frac{1}{3 k^{2}+1}\left[-2 \delta r+\frac{k^{2} r^{2}}{R_{0}}-\left.4 r^{2} R_{0} q_{0}^{2} \frac{d \beta}{d\left(r^{2}\right)}\right|_{r=0}\right] .
$$

After substituting Eqs. (B19)-(B22) and (B24) for $X_{(1,2)}, Y_{(1,2)}, Z_{(2)}, B_{\varphi(0)}, B_{\theta(2)}$, and $B_{\varphi(2)}$, the second order of Eq. (B15) yields:

$$
\begin{aligned}
B_{(2)} & =B_{(0)}\left\{-\frac{\beta_{(2)}}{2}-\frac{k \iota_{(0)}^{2}}{B_{(0)} R_{0}}+\frac{X_{(2) 0}-X_{(2) c}}{R_{0}}\right. \\
& \left.+\left[\frac{2}{k B_{(0)} R_{0}^{2}}+\frac{\left(k-k^{-1}\right) \iota_{(0)}^{2}}{B_{(0)} R_{0}}+2 \frac{X_{(2) c}}{R_{0}}\right] \cos ^{2}(\theta)\right\} .
\end{aligned}
$$

The formulae for $B$ used in our paper [Eqs. (16)-(18)] result from Eqs. (B9), (B26), and (B36) after substituting Eq. (B33) for $X_{(2) 0}$ and Eq. (B34) for $X_{(2) c}$ and expressing $\psi$ in terms of $r$ and $d$ in terms of $\delta$. 


\section{REFERENCES}

${ }^{1}$ Ya. I. Kolesnichenko, V. S. Marchenko, and R. B. White, Phys. Plasmas 8, 3143 (2001)

${ }^{2}$ Ya. I. Kolesnichenko, V. V. Lutsenko, V.S. Marchenko, R. B. White, and Yu. V. Yakovenko, 19th International Atomic Energy Agency Fusion Energy Conference, Lyon, 2002, Book of Abstracts (International Atomic Energy Agency, 2002), p. 146, Rep. TH/P3-15.

${ }^{3}$ A. A. Galeev and R. Z. Sagdeev, Reviews of Plasma Physics (Consultants Bureau, New York, 1979), Vol. 7, p. 257.

${ }^{4}$ Ya. I. Kolesnichenko, V. V. Lutsenko, and V. S. Marchenko, Nucl. Fusion 40, 1731 (2000).

${ }^{5}$ A. H. Boozer, Phys. Fluids 24, 1999 (1981).

${ }^{6}$ J. D. Meiss and R. D. Hazeltine, Phys. Fluids B 2, 2563 (1990).

${ }^{7}$ R. B. White, The Theory of Toroidally Confined Plasmas (Imperial College Press, London, 2001).

${ }^{8}$ L. S. Solov'ev and V. D. Shafranov, Reviews of Plasma Physics (Consultants Bureau, New York, 1970), Vol. 5, p. 1.

${ }^{9}$ A.H. Boozer, Plasma Phys. Control. Fusion 37, A103 (1995).

${ }^{10}$ M. Ono, S. M. Kaye, Y.-K. M. Peng et al., Nucl. Fusion 40, 557 (2000).

${ }^{11}$ A. N. Kaufman, Phys. Fluids 15, 1063 (1972).

${ }^{12}$ R. G. Littlejohn, J. Plasma Phys. 29, 111 (1983).

${ }^{13}$ Ya. I. Kolesnichenko, V. V. Lutsenko, R. B. White, and Yu. V. Yakovenko, Phys. Plasmas 5, 2963 (1998).

${ }^{14}$ O. P. Pogutse and E. I. Yurchenko, Reviews of Plasma Physics (Consultants Bureau, New York, 1986), Vol. 11, p. 65. 
${ }^{15}$ Ya. I. Kolesnichenko, R. B. White, and Yu. V. Yakovenko, Phys. Plasmas 9, 2639 (2002).

${ }^{16}$ V. D. Shafranov and E. I. Yurchenko, Nucl. Fusion 8, 829 (1968).

${ }^{17}$ D. Lortz and J. Nührenberg, Z. Naturforsch. 31a, 1277 (1976); 34a, 167 (1979).

${ }^{18}$ D. A. Garren and A. H. Boozer, Phys. Fluids B 3, 2805 (1991).

${ }^{19}$ Ya. I. Kolesnichenko, V. V. Lutsenko, H. Wobig, Yu. V. Yakovenko, and O. P. Fesenyuk, Phys. Plasmas 8, 491 (2001).

${ }^{20}$ B. A. Dubrovin, S. P. Novikov, and A. T. Fomenko, Sovremennaya geometriya (Moscow, Nauka, 1979) [in Russian]. 


\section{External Distribution}

Plasma Research Laboratory, Australian National University, Australia

Professor I.R. Jones, Flinders University, Australia

Professor João Canalle, Instituto de Fisica DEQ/IF - UERJ, Brazil

Mr. Gerson O. Ludwig, Instituto Nacional de Pesquisas, Brazil

Dr. P.H. Sakanaka, Instituto Fisica, Brazil

The Librarian, Culham Laboratory, England

Mrs. S.A. Hutchinson, JET Library, England

Professor M.N. Bussac, Ecole Polytechnique, France

Librarian, Max-Planck-Institut für Plasmaphysik, Germany

Jolan Moldvai, Reports Library, MTA KFKI-ATKI, Hungary

Dr. P. Kaw, Institute for Plasma Research, India

Ms. P.J. Pathak, Librarian, Insitute for Plasma Research, India

Ms. Clelia De Palo, Associazione EURATOM-ENEA, Italy

Dr. G. Grosso, Instituto di Fisica del Plasma, Italy

Librarian, Naka Fusion Research Establishment, JAERI, Japan

Library, Plasma Physics Laboratory, Kyoto University, Japan

Research Information Center, National Institute for Fusion Science, Japan

Dr. O. Mitarai, Kyushu Tokai University, Japan

Library, Academia Sinica, Institute of Plasma Physics, People's Republic of China

Shih-Tung Tsai, Institute of Physics, Chinese Academy of Sciences, People's Republic of China

Dr. S. Mirnov, TRINITI, Troitsk, Russian Federation, Russia

Dr. V.S. Strelkov, Kurchatov Institute, Russian Federation, Russia

Professor Peter Lukac, Katedra Fyziky Plazmy MFF UK, Mlynska dolina F-2, Komenskeho Univerzita, SK-842 15 Bratislava, Slovakia

Dr. G.S. Lee, Korea Basic Science Institute, South Korea

Institute for Plasma Research, University of Maryland, USA

Librarian, Fusion Energy Division, Oak Ridge National Laboratory, USA

Librarian, Institute of Fusion Studies, University of Texas, USA

Librarian, Magnetic Fusion Program, Lawrence Livermore National Laboratory, USA

Library, General Atomics, USA

Plasma Physics Group, Fusion Energy Research Program, University of California at San Diego, USA

Plasma Physics Library, Columbia University, USA

Alkesh Punjabi, Center for Fusion Research and Training, Hampton University, USA

Dr. W.M. Stacey, Fusion Research Center, Georgia Institute of Technology, USA

Dr. John Willis, U.S. Department of Energy, Office of Fusion Energy Sciences, USA

Mr. Paul H. Wright, Indianapolis, Indiana, USA 
The Princeton Plasma Physics Laboratory is operated by Princeton University under contract with the U.S. Department of Energy.

\author{
Information Services \\ Princeton Plasma Physics Laboratory \\ P.O. Box 451 \\ Princeton, NJ 08543
}

Phone: 609-243-2750

Fax: 609-243-2751

e-mail: pppl_info@pppl.gov

Internet Address: http://www.pppl.gov 